OP22

\title{
Automatic Classification System of Raman Spectra Applied to Pigments Analysis
}

\section{J. J. González-Vidal, ${ }^{1,2}$ R. Pérez-Pueyo, ${ }^{1}$ M. J. Soneira, ${ }^{1}$ S. Ruiz-Moreno ${ }^{1}$}

${ }^{1}$ Signal Theory and Communications Department, ETSETB, Universitat Politècnica de Catalunya, Sor Eulàlia d'Anzizu s/n, D5-101, Campus Nord, 08034, Barcelona (Spain), tel. +34 9340164 42, email: juanjo.glezvidal@tsc.upc.edu

2 Institut de Ciències del Cosmos - Universitat de Barcelona, Institut d'Estudis Espacials de Catalunya, Martí i Franquès 1, V705I, Facultat de Física, 08028, Barcelona (Spain), tel. +34 $93403 \quad 13$ 27, email: jjgonzalez@icc.ub.edu

Keywords: Raman spectroscopy, pigment classification, principal component analysis, multiple discriminant analysis

Raman spectroscopy has proved to be a powerful technique for the non-destructive characterization of constituent pigmentation in art works ${ }^{[1]}$. This task is important for the cataloguing, conservation and restoration of paintings. It is well known that Raman spectroscopy, being a spectroscopic method for molecular analysis, is able to discriminate between different molecular species based on the acquired Raman spectra. This discrimination is generally performed through visual comparison between Raman spectra measured on analyzed art works with an appropriate set of reference Raman spectra. In general, these reference spectra are measured from pigment powders under certain measurement conditions. However, pigments in works of art are usually mixed with other materials (such as binding agents, varnishes, other pigments, ...) so that a Raman spectrum measured directly on an analyzed art work can lose specific information due to different reasons. Indeed, these external agents may hamper the acquisition of the Raman spectra of the pigments and may hinder their interpretation. Moreover, measurement conditions may produce changes in the spectrum of a specific pigment which may lead to an erroneous identification ${ }^{[2]}$. Furthermore, certain pigments can be found in natural and synthetic forms as the ultramarine blue ${ }^{[3]}$ or in different polymorphs as the PB15 ${ }^{[4]}$. There is little difference between the Raman spectra of these pigments and therefore their automated analysis is a challenge. The discrimination between them, this is, between the natural and synthetic forms or between different polymorphs, is an important topic in preservation of art works because the pigments differ not only in their chemical and physical characteristics (such as stability, solubility and hue) but also appeared at different times on the paint market and thus they may be used as markers for dating and authenticating art works.

In the present work, several chemometric techniques were explored to develop an analytical method to automatically discriminate between Raman spectra that are very similar between them. Hence, a fully automatic classification system of Raman spectra of pigments is presented. The system is aimed to be a fully automatic tool to help the analyst in the complex process of pigments classification. Concretely, it is based on the chemometric techniques of principal component analysis (PCA) and multiple discriminant analysis (MDA), and a decision algorithm specifically developed to select the corresponding reference class with no user input. PCA deals with the whole set of input data without paying specific attention to any underlying class structure, whereas MDA deals directly with the discrimination between classes. In this work, PCA is used as a dimensionality reduction procedure which projects the input spectra onto an uncorrelated space, equivalent in information content, where the spectral differences are highlighted and redundancies are removed. The spectral expression obtained through the PCA projection is then used to obtain a discriminant space by means of MDA. This discriminant space minimizes the differences between spectra belonging to a same class while maximizing the inter-class differences. The combination of both chemometric techniques allows to obtain well-defined classes, providing a powerful way to achieve class separability so that it is possible to discriminate between very similar spectra in an automatic way (see Figure 1). 

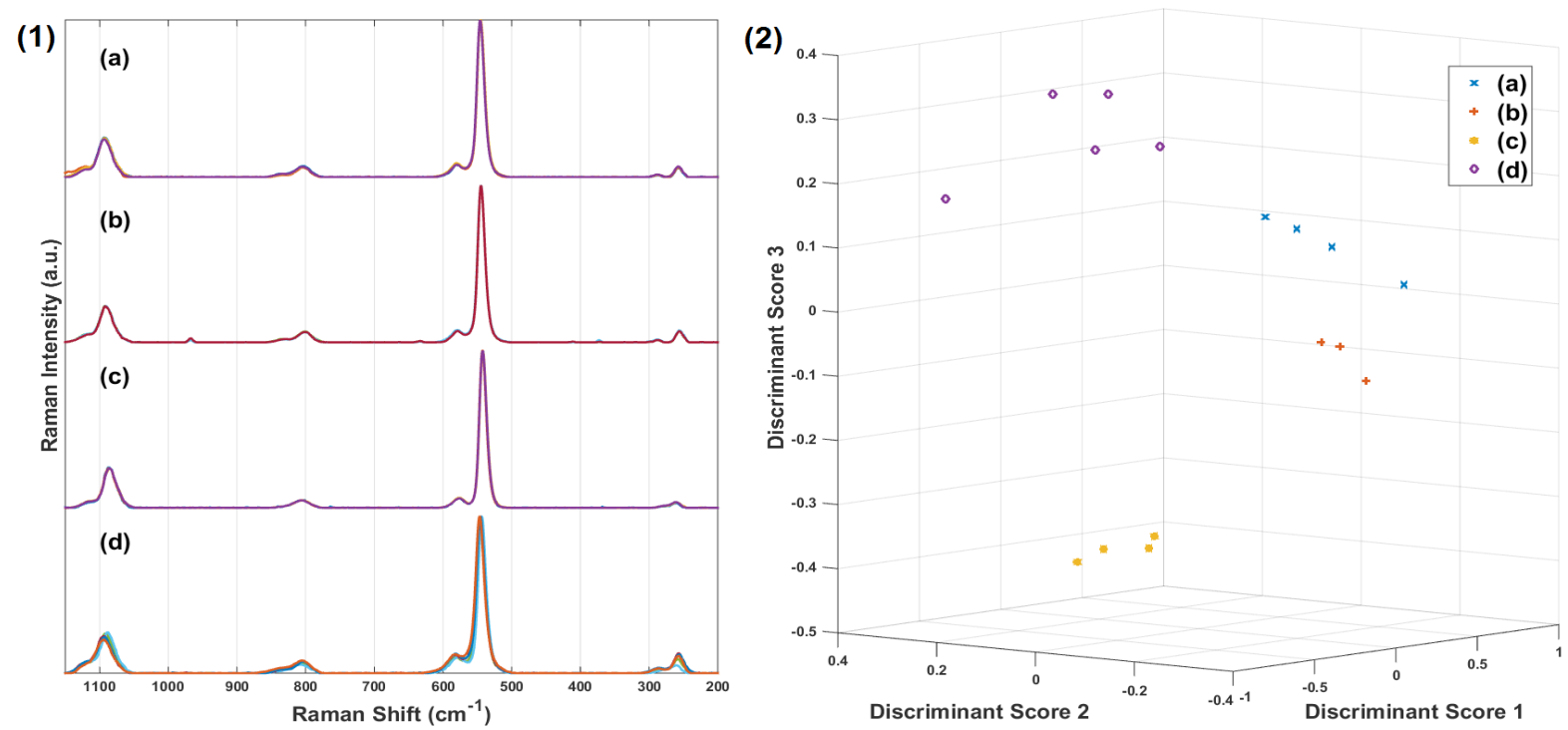

Figure 1. Example of differentiation between natural ultramarine blue from Afghanistan (a), Chile (b), and Siberia (c), and synthetic ultramarine blue (d): Raman spectra are shown in (1) and the discriminant projection obtained through the proposed methodology is shown in (2).

In order to diagnose the performance of the proposed methodology it was studied in a simulation stage simulating different practical problems commonly found in the spectral classification. The method was applied to the classification of artistic pigments in experimental cases, verifying the correct behavior of the implemented system. The reliable and consistent results that were obtained make the methodology a helpful tool suitable for the classification of pigments. Finally, although the proposed system has mainly been applied in pigment analysis, it could also be used in other fields to discriminate spectra that have very little differences.

\section{References}

[1] Chaplin T. D., Clark R. J. H., Singer B. W., J. Raman Spectrosc. 2014, 45, 1322-1325.

[2] Scherrer N.C., RAA2011: 6th International Congress on the Application of Raman Spectroscopy in Art and Archaeology, Parma (Italy), 5-8 September, 2011, Book of abstracts, p. 203-204.

[3] De Torres A. R., Ruiz-Moreno S., López-Gil A., Ferrer P., Chillón M. C., J. Raman Spectrosc. 2014, 45, 1279-1284.

[4] Defeyt, C., Vandenabeele, P., Gilbert, B., Van Pevenage, J., Cloots, R. and Strivay, D., J. Raman Spectrosc. 2012, 43, $1772-1780$. 